\title{
STABILITY OF THE MINIMAL SURFACE SYSTEM AND CONVEXITY OF AREA FUNCTIONAL
}

\author{
YNG-ING LEE AND MAO-PEI TSUI
}

\begin{abstract}
We study the convexity of the area functional for the graphs of maps with respect to the singular values of their differentials. Suppose that $f$ is a solution to the Dirichlet problem for the minimal surface system and the area functional is convex at $f$. Then the graph of $f$ is stable. New criteria for the stability of minimal graphs in any co-dimension are derived in the paper by this method. Our results in particular generalize the co-dimension one case, and improve the condition in the 2003 paper of the first author and M.-T. Wang from $\left|\bigwedge^{2} d f\right| \leq \frac{1}{p-1}$ to $\left|\bigwedge^{2} d f\right| \leq \frac{1}{\sqrt{p-1}}$, where $p$ is an upper bound of the rank of $d f$, and the condition in the 2008 paper of the first author and M.-T. Wang from $\sqrt{\operatorname{det}\left(I+(d f)^{T} d f\right)} \leq \frac{43}{40}$ to $\sqrt{\operatorname{det}\left(I+(d f)^{T} d f\right)} \leq 2$.
\end{abstract}

\section{INTRODUCTION}

Let $f=\left(f^{1}, \cdots, f^{m}\right): \Omega \rightarrow \mathbb{R}^{m}$ be a $C^{2}$ vector-valued function on $\Omega \subset \mathbb{R}^{n}$. Recall that $f=\left(f^{1}, \cdots, f^{m}\right): \Omega \rightarrow \mathbb{R}^{m}$ is said to be a solution to the minimal surface system (see Osserman [OS] or Lawson-Osserman [LO]) if

$$
\sum_{i, j=1}^{n} \frac{\partial}{\partial x^{i}}\left(\sqrt{g} g^{i j} \frac{\partial f^{\alpha}}{\partial x^{j}}\right)=0 \quad \text { for each } \alpha=1, \cdots, m,
$$

where $g_{i j}=\delta_{i j}+\sum_{\alpha} \frac{\partial f^{\alpha}}{\partial x^{i}} \frac{\partial f^{\alpha}}{\partial x^{j}}, g=\operatorname{det}_{i j}$ and $g^{i j}$ is the $(i, j)$ entry of the inverse matrix of $\left(g_{i j}\right)$. The graph of $f$ is called a non-parametric minimal submanifold. Equation (1.1) is indeed the Euler-Lagrange equation of the area functional $\int_{\Omega} \sqrt{g} d x^{1} \wedge \cdots \wedge d x^{n}$.

In the co-dimension one case, i.e. $m=1$, equation (1.1) is equivalent to the following equation:

$$
\operatorname{div}\left(\frac{\nabla f}{\sqrt{1+|\nabla f|^{2}}}\right)=0
$$

It is well known that the solution to (1.2) subject to the Dirichlet boundary condition is unique and stable (see, for example, Lawson-Osserman [LO]). This result follows easily from the convexity of the area functional. However, both uniqueness and stability for the solution to the minimal surface system do not hold in higher co-dimension in general. A counterexample was constructed by Lawson and Osserman in [LO] when $n=m=2$.

In higher co-dimension, the uniqueness and stability of the minimal surface systems were studied in [LW1] and [LW2]. A key ingredient in their works is a new expression of the second variation formula in terms of the singular values of $d f$.

Received by the editors November 23, 2011.

2010 Mathematics Subject Classification. Primary 53A10. 
In this paper, we first derive the convexity condition for the area functional with respect to its associated singular values in higher co-dimension. Then we prove that the solution to the minimal surface system is stable whenever the area functional is convex at the graph. We also obtain several criteria for the stability of minimal graphs in terms of the singular values of $d f$. These results are the direct application of convexity of the area functional. To describe the results, we recall the definition of the singular values of $d f$.

Let $f$ be a smooth map from $(M, g)$ to $(N, h), d f$ be the differential of $f$ and $(d f)^{T}$ be the adjoint of $d f$. The graph of $f$ is an embedded submanifold $\Sigma$ in the product manifold $\mathfrak{M}=M \times N$. Denote the singular values of $d f$, or eigenvalues of $\sqrt{(d f)^{T} d f}$, by $\left\{\lambda_{i}\right\}_{i=1}^{n}$. We prove the following stability theorem.

Theorem A (see Theorem4.1). Let $M$ and $N$ be Riemannian manifolds and $\Sigma$ be the graph of a map $f: M \rightarrow N$. Suppose the sectional curvature of $N$ is non-positive and $\Sigma$ is minimal in $M \times N$. Then $\Sigma$ is stable if $\lambda_{i}^{2} \lambda_{j}^{2} \leq 1$ for any $1 \leq i \neq j \leq n$, an

$$
1+\sum_{k=2}^{n}(k-1)(-1)^{k-1} \sum_{1 \leq j_{1}<j_{2}<\cdots<j_{k} \leq n} \lambda_{j_{1}}^{2} \cdots \lambda_{j_{k}}^{2} \geq 0
$$

or equivalently

$$
\prod_{i=1}^{n}\left(1-\lambda_{i}^{2}\right)+\sum_{i=1}^{n}\left(1-\lambda_{1}^{2}\right) \cdots \lambda_{i}^{2} \cdots\left(1-\lambda_{n}^{2}\right) \geq 0 .
$$

The conditions in Theorem A correspond to the convexity of the area functional, and the theorem in particular covers the co-dimension one case. We also prove a stability theorem in terms of the two Jacobians of $f$. To describe the result, we first recall some notation. Let $L: \mathbb{R}^{n} \rightarrow \mathbb{R}^{m}$ be a linear transformation. It induces a linear transformation $\bigwedge^{2} L$, from the wedge product $\bigwedge^{2} \mathbb{R}^{n}$ to $\bigwedge^{2} \mathbb{R}^{m}$ by

$$
\left(\bigwedge^{2} L\right)(v \wedge w)=L(v) \wedge L(w) .
$$

With this we define

$$
\left|\bigwedge^{2} L\right|=\sup _{|v \wedge w|=1}\left|\left(\bigwedge^{2} L\right)(v \wedge w)\right| .
$$

In particular, $\left|\bigwedge^{2} L\right|=0$ if $L$ is of rank one. Let $f: \Omega \subset \mathbb{R}^{n} \rightarrow \mathbb{R}^{m}$ and $d f: \Omega \subset$ $\mathbb{R}^{n} \rightarrow \mathbb{R}^{m}$. We define $\left|\bigwedge^{2} d f\right|=\sup _{x \in \Omega}\left|\bigwedge^{2} d f(x)\right|$. Note that

$$
\left|\bigwedge^{2} d f\right|=\sup _{x \in \Omega, 1 \leq i<j \leq n} \lambda_{i}(x) \lambda_{j}(x),
$$

where $\left\{\lambda_{k}(x)\right\}_{k=1}^{n}$ are the singular values of $d f(x)$.

Theorem B (see Theorem 4.5). Suppose that a non-parametric minimal submanifold $\Sigma$ is the graph of a map $f: \Omega \subset \mathbb{R}^{n} \rightarrow \mathbb{R}^{m}$. Then $\Sigma$ is stable if $\left|\bigwedge^{2} d f\right| \leq \frac{1}{\sqrt{n-1}}$.

A more general version of the theorem is proved in Theorem 4.5. We prove the result for minimal maps between Riemannian manifolds and have the bound in terms of the rank of $d f$. $f$.

Our last theorem on stability is in terms of the magnitude of the differential of 
Theorem C (see Theorem 4.6). Suppose that a non-parametric minimal submanifold $\Sigma$ is the graph of a map $f: \Omega \subset \mathbb{R}^{n} \rightarrow \mathbb{R}^{m}$. Then $\Sigma$ is stable if $\operatorname{det}\left(I+\left(d f^{T}\right) d f\right) \leq 4$.

Theorem B generalizes Theorem 4.1 in [LW2] and Theorem C generalizes Theorem $\mathrm{A}$ in [LW1.

\section{BACKGROUND AND NOTATION}

In this section, we recall some notation and formulae that will be used later. Suppose that $(M, g)$ and $(N, h)$ are two Riemannian manifolds with dimensions $n$ and $m$ respectively. Let $f$ be a smooth map from $(M, g)$ to $(N, h), d f$ be the differential of $f$ and $(d f)^{T}$ be the adjoint of $d f$. The graph of $f$ is an embedded submanifold $\Sigma$ in the product manifold $\mathfrak{M}=M \times N$. Denote the projections by $\pi_{1}: \mathfrak{M} \rightarrow M$ and $\pi_{2}: \mathfrak{M} \rightarrow N$. For simplicity, we still denote the differentials by $\pi_{1}: T_{q} \mathfrak{M} \rightarrow T_{\pi_{1}(q)} M$ and $\pi_{2}: T_{q} \mathfrak{M} \rightarrow T_{\pi_{2}(q)} N$ at any point $q \in \mathfrak{M}$.

Next, we introduce the singular values of $d f$ and special bases adapted to $d f$. Denote the singular values of $d f$, or eigenvalues of $\sqrt{(d f)^{T} d f}$, by $\left\{\lambda_{i}\right\}_{i=1}^{n}$. Let $p$ denote the rank of $d f$. We can rearrange them so that $\lambda_{i}=0$ for $i \geq p+1$. By singular value decomposition, there exist orthonormal bases

$$
\left\{a_{i}\right\}_{i=1}^{n} \text { for } T_{\pi_{1}(q)} M \text { and }\left\{a_{\alpha}\right\}_{\alpha=n+1}^{n+m} \text { for } T_{\pi_{2}(q)} N
$$

for $q \in \Sigma$ such that

$$
d f\left(a_{i}\right)=\lambda_{i} a_{n+i}
$$

for $i$ less than or equal to $p$ and $d f\left(a_{i}\right)=0$ for $i$ greater than $p$. Moreover,

$$
e_{i}= \begin{cases}\frac{1}{\sqrt{1+\lambda_{i}^{2}}}\left(a_{i}+\lambda_{i} a_{n+i}\right) & \text { if } 1 \leq i \leq p \\ a_{i} & \text { if } p+1 \leq i \leq n\end{cases}
$$

becomes an orthonormal basis for the tangent space $T_{q} \Sigma$ and

$$
e_{n+j}= \begin{cases}\frac{1}{\sqrt{1+\lambda_{j}^{2}}}\left(a_{n+j}-\lambda_{j} a_{j}\right) & \text { if } 1 \leq j \leq p, \\ a_{n+j} & \text { if } p+1 \leq j \leq m\end{cases}
$$

becomes an orthonormal basis for the normal space $N_{q} \Sigma$. We also denote

$$
b_{j}=a_{n+j} \text { for } 1 \leq j \leq m .
$$

We first recall the non-parametric variation formula of area for graphs derived in LW2. Fix a local coordinate system $\left\{x^{i}\right\}$ on $M$. Then the induced metric on $\Sigma$ is given by

$$
\sum_{i, j=1}^{n} G_{i j} d x^{i} d x^{j}=\sum_{i, j=1}^{n}\left(g_{i j}+\left\langle d f\left(\frac{\partial}{\partial x^{i}}\right), d f\left(\frac{\partial}{\partial x^{j}}\right)\right\rangle\right) d x^{i} d x^{j},
$$

and the area of the graph is

$$
\mathcal{V}=\int_{M} \sqrt{\operatorname{det} G_{i j}} d x^{1} \wedge \cdots \wedge d x^{n}=\int_{M} \sqrt{\prod_{i=1}^{n}\left(1+\lambda_{i}^{2}\right)} d x^{1} \wedge \cdots \wedge d x^{n}=\int_{M} d v
$$


Note that

$$
\sqrt{\operatorname{det} G_{i j}}=\sqrt{\prod_{i=1}^{n}\left(1+\lambda_{i}^{2}\right)}
$$

if we choose $\frac{\partial}{\partial x^{i}}=a_{i}$, which is defined in (2.1) and (2.2). Assume that there is a family of maps $f_{t}, 0 \leq t \leq \epsilon$, from $M$ to $N$ with $f_{0}=f$ on $M$ and $f_{t}=f$ outside a compact subset of $M$. When the boundary of $M$ is non-empty, we require that $f_{t}=f$ on $\partial M$. Let $G^{i j}(t)$ be the $(i, j)$ entry of the inverse matrix of $\left(G_{i j}(t)\right)$. Denote the variation field $\frac{d f_{t}}{d t}$ by $V(t)$. For simplicity, we will omit the dependency of $G_{i j}$ and $V$ on $t$.

The following proposition gives a lower bound of the second variation formula of area in terms of the singular values and singular vectors which is derived in (4.2) of [LW2].

Proposition 1 ([LW2 $)$. Let $(M, g)$ and $(N, h)$ be Riemannian manifolds and $\Sigma$ be the graph of a map $f: M \rightarrow N$ with $\operatorname{rank}(d f) \leq p$ for some integer $p>1$. Suppose the sectional curvature of $N$ is non-positive and $\Sigma$ is minimal in $M \times N$. Then the second variation formula of area satisfies the following inequality:

$$
\begin{aligned}
\left.\frac{d^{2} \mathcal{V}_{t}}{d t^{2}}\right|_{t=0} \geq & \int_{M}\left(\sum_{i} \frac{1}{\left(1+\lambda_{i}^{2}\right)^{2}}\left\langle\nabla_{d f\left(a_{i}\right)} V, b_{i}\right\rangle^{2}\right. \\
& \left.+\sum_{i \neq j} \frac{\lambda_{i} \lambda_{j}}{\left(1+\lambda_{i}^{2}\right)\left(1+\lambda_{j}^{2}\right)}\left\langle\nabla_{d f\left(a_{i}\right)} V, b_{i}\right\rangle\left\langle\nabla_{d f\left(a_{j}\right)} V, b_{j}\right\rangle\right) d v \\
& +\int_{M}\left(\sum_{i \neq j} \frac{1}{\left(1+\lambda_{i}^{2}\right)\left(1+\lambda_{j}^{2}\right)}\left\langle\nabla_{d f\left(a_{i}\right)} V, b_{j}\right\rangle^{2}\right. \\
& \left.-\sum_{i \neq j} \frac{\lambda_{i} \lambda_{j}}{\left(1+\lambda_{i}^{2}\right)\left(1+\lambda_{j}^{2}\right)}\left\langle\nabla_{d f\left(a_{i}\right)} V, b_{j}\right\rangle\left\langle\nabla_{d f\left(a_{j}\right)} V, b_{i}\right\rangle\right) d v .
\end{aligned}
$$

Here the inner product and the connection $\nabla$ are with respect to the metric $h$ on $N$.

Now, we recall some basic facts that will be used later.

Lemma 2.1. For any $v=\left(v_{1}, \cdots, v_{n}\right)$, we have

(i) $\operatorname{det}\left(I+v^{T} v\right)=1+\sum_{i=1}^{n} v_{i}^{2}$ and

(ii) $\operatorname{det}\left(D+v^{T} v\right)=\prod_{i=1}^{n} d_{i}+\sum_{i=1}^{n} d_{1} \cdots d_{i-1} v_{i}^{2} d_{i+1} \cdots d_{n}$, where $D=$ $\operatorname{diag}\left(d_{1}, \cdots, d_{n}\right)$.

Proof. The first equality follows from the fact that the matrix $A=I+v^{T} v$ has eigenvectors $v^{T}$ and $\left(v^{T}\right)^{\perp}$ whose eigenvalues are $1+\sum_{i=1}^{n} v_{i}^{2}$ and 1 .

For (ii), we first assume that $D$ is non-singular and denote $D^{\frac{1}{2}}=\operatorname{diag}\left(\sqrt{d_{1}}, \cdots\right.$, $\left.\sqrt{d_{n}}\right)$. Note that $d_{i}$ is allowed to be negative valued and $v$ can be a complex vector. Since

we have

$$
D+v^{T} v=D^{\frac{1}{2}}\left(I+\left(v D^{-\frac{1}{2}}\right)^{T}\left(v D^{-\frac{1}{2}}\right)\right) D^{\frac{1}{2}}
$$

$$
\begin{aligned}
& \operatorname{det}\left(D+v^{T} v\right)=\operatorname{det}(D) \operatorname{det}\left(I+\left(v D^{-\frac{1}{2}}\right)^{T}\left(v D^{-\frac{1}{2}}\right)\right) \\
= & \operatorname{det}(D)\left(1+\sum_{i=1}^{n} \frac{v_{i}^{2}}{d_{i}}\right)=\prod_{i=1}^{n} d_{i}+\sum_{i=1}^{n} d_{1} \cdots d_{i-1} v_{i}^{2} d_{i+1} \cdots d_{n} .
\end{aligned}
$$


If $D$ is singular, we can obtain this result by taking the limit of non-singular approximation.

Lemma 2.2. The inequality

$$
\left|\lambda_{i} \lambda_{j}\right| \leq-1+\sqrt{\prod_{i=1}^{n}\left(1+\lambda_{i}^{2}\right)}
$$

holds for $1 \leq i \neq j \leq n$.

Proof. It follows from the inequality

$$
\prod_{i=1}^{n}\left(1+\lambda_{i}^{2}\right) \geq\left(1+\lambda_{i}^{2}\right)\left(1+\lambda_{j}^{2}\right)=1+\lambda_{i}^{2}+\lambda_{j}^{2}+2 \lambda_{i}^{2} \lambda_{j}^{2} \geq\left(1+\left|\lambda_{i} \lambda_{j}\right|\right)^{2}
$$

for $1 \leq i \neq j \leq n$.

\section{Convexity of the area functional}

Recall that we can write the area functional in terms of the singular values as in (2.7). In this section, we will derive the convexity condition for the area functional with respect to the singular values.

Lemma 3.1. The area functional $A=\prod_{k=1}^{n}\left(1+\lambda_{k}^{2}\right)^{\frac{1}{2}}$ is convex if and only if the quadratic form

$$
\sum_{i} \frac{w_{i}^{2}}{\left(1+\lambda_{i}^{2}\right)^{2}}+\sum_{i \neq j} \frac{\lambda_{i} \lambda_{j} w_{i} w_{j}}{\left(1+\lambda_{i}^{2}\right)\left(1+\lambda_{j}^{2}\right)} \geq 0
$$

for any $w=\left(w_{1}, \cdots, w_{n}\right)$.

Proof. Denote $F=\ln A=\sum_{k=1}^{n} \frac{\ln \left(1+\lambda_{k}^{2}\right)}{2}$. Then

$$
\frac{\partial F}{\partial \lambda_{i}}=\frac{\lambda_{i}}{1+\lambda_{i}^{2}} \quad \text { and } \quad \frac{\partial^{2} F}{\partial \lambda_{i} \lambda_{j}}=\frac{1-\lambda_{i}^{2}}{\left(1+\lambda_{i}^{2}\right)^{2}} \delta_{i j}
$$

A simple calculation shows that

$$
\frac{\partial F}{\partial \lambda_{i}}=\frac{\partial A}{\partial \lambda_{i}} \frac{1}{A}, \quad \frac{\partial^{2} F}{\partial \lambda_{i} \partial \lambda_{j}}=\frac{\partial^{2} A}{\partial \lambda_{i} \partial \lambda_{j}} \frac{1}{A}-\frac{\partial A}{\partial \lambda_{i}} \frac{\partial A}{\partial \lambda_{j}} \frac{1}{A^{2}}
$$

and

$$
\frac{\partial^{2} A}{\partial \lambda_{i} \partial \lambda_{j}}=A\left(\frac{\partial^{2} F}{\partial \lambda_{i} \partial \lambda_{j}}+\frac{\partial A}{\partial \lambda_{i}} \frac{\partial A}{\partial \lambda_{j}} \frac{1}{A^{2}}\right)=A\left(\frac{1-\lambda_{i}^{2}}{\left(1+\lambda_{i}^{2}\right)^{2}} \delta_{i j}+\frac{\lambda_{i} \lambda_{j}}{\left(1+\lambda_{i}^{2}\right)\left(1+\lambda_{i}^{2}\right)}\right) .
$$

Let $\mathcal{A}=\operatorname{Hess}(A)=A\left(D+v^{T} v\right)$, where $D=\operatorname{diag}\left(\frac{1-\lambda_{1}^{2}}{\left(1+\lambda_{1}^{2}\right)^{2}}, \cdots, \frac{1-\lambda_{n}^{2}}{\left(1+\lambda_{n}^{2}\right)^{2}}\right)$ and $v=\left(\frac{\lambda_{1}}{1+\lambda_{1}^{2}}, \cdots, \frac{\lambda_{n}}{1+\lambda_{n}^{2}}\right)$. This lemma follows from the fact that the quadratic form 
associated with $\mathcal{A}$ is

$$
\begin{aligned}
w^{T} \mathcal{A} w & =\sum_{i, j} w_{i} w_{j} \frac{\partial^{2} A}{\partial \lambda_{i} \partial \lambda_{j}} \\
& =A\left(\sum_{i} \frac{1-\lambda_{i}^{2}}{\left(1+\lambda_{i}^{2}\right)^{2}} w_{i}^{2}+\sum_{i, j} \frac{\lambda_{i} \lambda_{j} w_{i} w_{j}}{\left(1+\lambda_{i}^{2}\right)\left(1+\lambda_{j}^{2}\right)}\right) \\
& =A\left(\sum_{i}\left(\frac{\left(1-\lambda_{i}^{2}\right)}{\left(1+\lambda_{i}^{2}\right)^{2}} w_{i}^{2}+\frac{\lambda_{i}^{2} w_{i}^{2}}{\left(1+\lambda_{i}^{2}\right)^{2}}\right)+\sum_{i \neq j} \frac{\lambda_{i} \lambda_{j} w_{i} w_{j}}{\left(1+\lambda_{i}^{2}\right)\left(1+\lambda_{j}^{2}\right)}\right) \\
& =A\left(\sum_{i} \frac{w_{i}^{2}}{\left(1+\lambda_{i}^{2}\right)^{2}}+\sum_{i \neq j} \frac{\lambda_{i} \lambda_{j} w_{i} w_{j}}{\left(1+\lambda_{i}^{2}\right)\left(1+\lambda_{j}^{2}\right)}\right) .
\end{aligned}
$$

Next, we derive an equivalent condition for the convexity of the area functional which will be used later.

Theorem 3.2. The area functional $A=\prod_{k=1}^{n}\left(1+\lambda_{k}^{2}\right)^{\frac{1}{2}}$ is convex if and only if $\lambda_{i}^{2} \lambda_{j}^{2} \leq 1$ for any $1 \leq i \neq j \leq n$ and

$$
\prod_{i=1}^{n}\left(1-\lambda_{i}^{2}\right)+\sum_{i=1}^{n}\left(1-\lambda_{1}^{2}\right) \cdots \lambda_{i}^{2} \cdots\left(1-\lambda_{n}^{2}\right) \geq 0 .
$$

Proof. Recall that the hessian of the area functional is $\mathcal{A}=A\left(D+v^{T} v\right)$, where $D=\operatorname{diag}\left(\frac{1-\lambda_{1}^{2}}{\left(1+\lambda_{1}^{2}\right)^{2}}, \cdots, \frac{1-\lambda_{n}^{2}}{\left(1+\lambda_{n}^{2}\right)^{2}}\right)$ and $v=\left(\frac{\lambda_{1}}{1+\lambda_{1}^{2}}, \cdots, \frac{\lambda_{n}}{1+\lambda_{n}^{2}}\right)$. A direct computation gives $\mathcal{A}_{i i}=\frac{A}{\left(1+\lambda_{i}^{2}\right)^{2}}>0$ and $\mathcal{A}_{i i} \mathcal{A}_{j j}-\mathcal{A}_{i j}^{2}=\frac{A^{2}\left(1-\lambda_{i}^{2} \lambda_{j}^{2}\right)}{\left(1+\lambda_{i}^{2}\right)^{2}\left(1+\lambda_{j}^{2}\right)^{2}}$ for $i \neq j$. Let $\mathcal{A}_{k}$ be the $k \times k$ principle submatrix of the matrix $\mathcal{A}$. Using Lemma 2.1, we get

$$
\begin{aligned}
\operatorname{det}\left(\mathcal{A}_{k}\right) & =A^{k}\left(\prod_{i=1}^{k} \frac{1-\lambda_{i}^{2}}{\left(1+\lambda_{i}^{2}\right)^{2}}+\sum_{i=1}^{k} \frac{1-\lambda_{1}^{2}}{\left(1+\lambda_{1}^{2}\right)^{2}} \cdots \frac{\lambda_{i}^{2}}{\left(1+\lambda_{i}^{2}\right)^{2}} \cdots \frac{1-\lambda_{k}^{2}}{\left(1+\lambda_{k}^{2}\right)^{2}}\right) \\
& =A^{k}\left(\frac{\prod_{i=1}^{k}\left(1-\lambda_{i}^{2}\right)+\sum_{i=1}^{k}\left(1-\lambda_{1}^{2}\right) \cdots \lambda_{i}^{2} \cdots\left(1-\lambda_{k}^{2}\right)}{\prod_{i=1}^{k}\left(1+\lambda_{i}^{2}\right)^{2}}\right)
\end{aligned}
$$

and

$$
\operatorname{det}(\mathcal{A})=A^{n}\left(\frac{\prod_{i=1}^{n}\left(1-\lambda_{i}^{2}\right)+\sum_{i=1}^{n}\left(1-\lambda_{1}^{2}\right) \cdots \lambda_{i}^{2} \cdots\left(1-\lambda_{n}^{2}\right)}{\prod_{i=1}^{n}\left(1+\lambda_{i}^{2}\right)^{2}}\right) .
$$

Suppose $A$ is convex. Then $\mathcal{A}_{i i} \mathcal{A}_{j j}-\mathcal{A}_{i j}^{2} \geq 0$ and $\operatorname{det}(\mathcal{A}) \geq 0$. It implies that $\lambda_{i}^{2} \lambda_{j}^{2} \leq 1$ for any $1 \leq i \neq j \leq n$ and (3.2) holds.

Now assume that $\lambda_{i}^{2} \lambda_{j}^{2} \leq 1$ for any $1 \leq i \neq j \leq n$ and (3.2) holds. We can order the singular values such that $0 \leq \lambda_{1} \leq \cdots \leq \lambda_{n-1} \leq \lambda_{n}$. Since $\lambda_{i}^{2} \lambda_{j}^{2} \leq 1$ for all $1 \leq i \neq j \leq n$, we have $0 \leq \lambda_{1} \leq \cdots \leq \lambda_{n-1} \leq 1$. Thus we have $\operatorname{det}\left(\mathcal{A}_{k}\right) \geq 0$ for $1 \leq k \leq n-1$ from (3.3) and $\operatorname{det}(\mathcal{A}) \geq 0$ from (3.2). Therefore $A$ is convex by the standard convexity test.

We have the following corollary immediately from the previous theorem.

Corollary 1. The area functional $A=\prod_{k=1}^{n}\left(1+\lambda_{k}^{2}\right)^{\frac{1}{2}}$ is convex if $\lambda_{i}^{2} \leq 1$ for any $1 \leq i \leq n$. 
Remark 3.3. From now on, we order the singular values so that

$$
0 \leq \lambda_{1} \leq \cdots \leq \lambda_{n-1} \leq \lambda_{n}
$$

Under the condition $\lambda_{i}^{2} \lambda_{j}^{2} \leq 1$ for any $1 \leq i \neq j \leq n$, we have either $\lambda_{i} \leq 1$ for $1 \leq i \leq n$, which implies the convexity of $A$ from Corollary 1 or

$$
0 \leq \lambda_{1} \leq \cdots \leq \lambda_{n-1}<1<\lambda_{n}
$$

Next, we derive an explicit formula for $(3.2)$ to get the following theorem.

Theorem 3.4. The area functional $A=\prod_{k=1}^{n}\left(1+\lambda_{k}^{2}\right)^{\frac{1}{2}}$ is convex if and only if $\lambda_{i}^{2} \lambda_{j}^{2} \leq 1$ for any $1 \leq i \neq j \leq n$ and

$$
1+\sum_{k=2}^{n}(k-1)(-1)^{k-1} \sum_{1 \leq j_{1}<j_{2}<\cdots<j_{k} \leq n} \lambda_{j_{1}}^{2} \cdots \lambda_{j_{k}}^{2} \geq 0 .
$$

Proof. We will show that

$$
\begin{aligned}
& \prod_{i=1}^{n}\left(1-\lambda_{i}^{2}\right)+\sum_{i=1}^{n}\left(1-\lambda_{1}^{2}\right) \cdots \lambda_{i}^{2} \cdots\left(1-\lambda_{n}^{2}\right) \\
= & 1+\sum_{k=2}^{n}(k-1)(-1)^{k-1} \sum_{1 \leq j_{1}<j_{2}<\cdots<j_{k} \leq n} \lambda_{j_{1}}^{2} \cdots \lambda_{j_{k}}^{2}
\end{aligned}
$$

by induction.

When $n=2$, we have

$$
\left(1-\lambda_{1}^{2}\right)\left(1-\lambda_{2}^{2}\right)+\lambda_{1}^{2}\left(1-\lambda_{2}^{2}\right)+\left(1-\lambda_{1}^{2}\right) \lambda_{2}^{2}=1-\lambda_{1}^{2} \lambda_{2}^{2} .
$$

Hence (3.7) holds. Suppose that equation (3.7) is true for $n=m$. When $n=m+1$, we can group the left hand side of (3.8) into terms involving $\lambda_{m+1}$ and those not involving $\lambda_{m+1}$. It gives

$$
\begin{aligned}
& \prod_{i=1}^{m+1}\left(1-\lambda_{i}^{2}\right)+\sum_{i=1}^{m+1}\left(1-\lambda_{1}^{2}\right) \cdots \lambda_{i}^{2} \cdots\left(1-\lambda_{m+1}^{2}\right) \\
= & \left(1-\lambda_{m+1}^{2}\right)\left(\prod_{i=1}^{m}\left(1-\lambda_{i}^{2}\right)+\sum_{i=1}^{m}\left(1-\lambda_{1}^{2}\right) \cdots \lambda_{i}^{2} \cdots\left(1-\lambda_{m}^{2}\right)\right)+\lambda_{m+1}^{2} \prod_{i=1}^{m}\left(1-\lambda_{i}^{2}\right) .
\end{aligned}
$$

Plugging the formula for $n=m$ into the first term of (3.8) and simplifying the second term, we have that (3.8) becomes

$$
\begin{aligned}
& \left(1-\lambda_{m+1}^{2}\right)\left(1+\sum_{k=2}^{m}(k-1)(-1)^{k-1} \sum_{1 \leq j_{1}<j_{2}<\cdots<j_{k} \leq m} \lambda_{j_{1}}^{2} \cdots \lambda_{j_{k}}^{2}\right) \\
& +\lambda_{m+1}^{2}\left(1+\sum_{k=1}^{m}(-1)^{k} \sum_{1 \leq j_{1}<j_{2}<\cdots<j_{k} \leq m} \lambda_{j_{1}}^{2} \cdots \lambda_{j_{k}}^{2}\right) .
\end{aligned}
$$


Expanding the previous expression, we obtain

$$
\begin{aligned}
& 1+\sum_{k=2}^{m}(k-1)(-1)^{k-1} \sum_{1 \leq j_{1}<j_{2}<\cdots<j_{k} \leq m} \lambda_{j_{1}}^{2} \cdots \lambda_{j_{k}}^{2} \\
& +\sum_{k=2}^{m}(k-1)(-1)^{k} \sum_{1 \leq j_{1}<j_{2}<\cdots<j_{k} \leq m} \lambda_{j_{1}}^{2} \cdots \lambda_{j_{k}}^{2} \lambda_{m+1}^{2} \\
& +\sum_{k=1}^{m}(-1)^{k} \sum_{1 \leq j_{1}<j_{2}<\cdots<j_{k} \leq m} \lambda_{j_{1}}^{2} \cdots \lambda_{j_{k}}^{2} \lambda_{m+1}^{2},
\end{aligned}
$$

which can be grouped as

$$
\begin{array}{rl} 
& 1+\sum_{k=2}^{m}(k-1)(-1)^{k-1} \sum_{1 \leq j_{1}<j_{2}<\cdots<j_{k} \leq m} \lambda_{j_{1}}^{2} \cdots \lambda_{j_{k}}^{2} \\
+ & \sum_{k=2}^{m+1} \sum_{1 \leq j_{1}<\cdots<j_{k-1} \leq m}\left((k-2)(-1)^{k-1} \lambda_{j_{1}}^{2} \cdots \lambda_{j_{k-1}}^{2} \lambda_{m+1}^{2}\right. \\
& \left.+(-1)^{k-1} \lambda_{j_{1}}^{2} \cdots \lambda_{j_{k-1}}^{2} \lambda_{m+1}^{2}\right) \\
= & 1+\sum_{k=2}^{m}(k-1)(-1)^{k-1} \sum_{1 \leq j_{1}<j_{2}<\cdots<j_{k} \leq m} \lambda_{j_{1}}^{2} \cdots \lambda_{j_{k}}^{2} \\
+\sum_{k=2}^{m+1} \sum_{1 \leq j_{1}<\cdots<j_{k-1} \leq m}^{(k-1)(-1)^{k-1} \lambda_{j_{1}}^{2} \cdots \lambda_{j_{k-1}}^{2} \lambda_{m+1}^{2}} & 1+\sum_{k=2}^{m+1}(k-1)(-1)^{k-1} \sum_{1 \leq j_{1}<j_{2}<\cdots<j_{k} \leq m+1} \lambda_{j_{1}}^{2} \cdots \lambda_{j_{k}}^{2} .
\end{array}
$$

Thus (3.7) holds for all $n \geq 2$.

We can use the previous theorem to find some special subsets of (3.6), which are easier to check.

Proposition 2. The area functional $A=\prod_{l=1}^{n}\left(1+\lambda_{l}^{2}\right)^{\frac{1}{2}}$ is convex if

$$
\lambda_{i}^{2} \lambda_{j}^{2} \leq 1 \text { for any } 1 \leq i \neq j \leq n
$$

and

$$
\left(\lambda_{i}^{2}+\lambda_{j}^{2}\right) \lambda_{k}^{2} \leq \frac{2}{n-1}+\left(2-\frac{2}{n-1}\right) \lambda_{i}^{2} \lambda_{j}^{2} \lambda_{k}^{2}
$$

for any $1 \leq i \neq j \neq k \leq n$.

Proof. Define

$$
\mathfrak{A}=\prod_{i=1}^{n}\left(1-\lambda_{i}^{2}\right)+\sum_{i=1}^{n}\left(1-\lambda_{1}^{2}\right) \cdots \lambda_{i}^{2} \cdots\left(1-\lambda_{n}^{2}\right) .
$$

From Theorem [3.2, it suffices to show that $\mathfrak{A}$ is non-negative when the condition (3.10) holds. Since $\lambda_{i}^{2} \lambda_{j}^{2} \leq 1$ for all $1 \leq i \neq j \leq n$, we only need to consider the case

$$
0 \leq \lambda_{1} \leq \cdots \leq \lambda_{n-1}<1<\lambda_{n}
$$


from Remark 3.3. Rewrite (3.11) as

$$
\begin{aligned}
& \prod_{i=1}^{n}\left(1-\lambda_{i}^{2}\right)\left[1+\sum_{k=1}^{n} \frac{\lambda_{k}^{2}}{1-\lambda_{k}^{2}}\right] \\
= & \prod_{i=1}^{n}\left(1-\lambda_{i}^{2}\right)\left[\frac{1}{1-\lambda_{n}^{2}}+\sum_{k=1}^{n-1} \frac{\lambda_{k}^{2}}{1-\lambda_{k}^{2}}\right] \\
= & \prod_{i=1}^{n-1}\left(1-\lambda_{i}^{2}\right)+\prod_{i=1}^{n}\left(1-\lambda_{i}^{2}\right) \cdot \frac{1}{2}\left[\sum_{j=1}^{n-2}\left(\frac{\lambda_{j}^{2}}{1-\lambda_{j}^{2}}+\frac{\lambda_{j+1}^{2}}{1-\lambda_{j+1}^{2}}\right)+\frac{\lambda_{1}^{2}}{1-\lambda_{1}^{2}}+\frac{\lambda_{n-1}^{2}}{1-\lambda_{n-1}^{2}}\right] .
\end{aligned}
$$

Using

$$
\begin{aligned}
\prod_{i=1}^{n-1}\left(1-\lambda_{i}^{2}\right)= & \sum_{j=1}^{n-2} \prod_{i \neq j, j+1}^{n-1}\left(1-\lambda_{i}^{2}\right) \frac{\left(1-\lambda_{j}^{2}\right)\left(1-\lambda_{j+1}^{2}\right)}{n-1} \\
& +\prod_{i \neq 1, n-1}^{n-1}\left(1-\lambda_{i}^{2}\right) \frac{\left(1-\lambda_{1}^{2}\right)\left(1-\lambda_{n-1}^{2}\right)}{n-1},
\end{aligned}
$$

equation (3.12) becomes

$$
\begin{aligned}
& \sum_{j=1}^{n-2}\left(\prod_{i \neq j, j+1}^{n-1}\left(1-\lambda_{i}^{2}\right)\right)\left[\frac{\left(1-\lambda_{j}^{2}\right)\left(1-\lambda_{j+1}^{2}\right)}{n-1}+\frac{\left(1-\lambda_{j+1}^{2}\right) \lambda_{j}^{2}\left(1-\lambda_{n}^{2}\right)}{2}+\frac{\left(1-\lambda_{j}^{2}\right) \lambda_{j+1}^{2}\left(1-\lambda_{n}^{2}\right)}{2}\right] \\
+ & \prod_{i \neq 1, n-1}^{n-1}\left(1-\lambda_{i}^{2}\right)\left[\frac{\left(1-\lambda_{1}^{2}\right)\left(1-\lambda_{n-1}^{2}\right)}{n-1}+\frac{\left.\left(1-\lambda_{1}^{2}\right) \lambda_{n-1}^{2}+\left(1-\lambda_{n-1}^{2}\right) \lambda_{1}^{2}\right)}{2} \cdot\left(1-\lambda_{n}^{2}\right)\right], \\
= & \sum_{j=1}^{n-2} \prod_{i \neq j, j+1}^{n-1}\left(1-\lambda_{i}^{2}\right) A_{j}+\prod_{i \neq 1, n-1}^{n-1}\left(1-\lambda_{i}^{2}\right) A_{n-1},
\end{aligned}
$$

where

$$
A_{j}=\frac{\left(1-\lambda_{j}^{2}\right)\left(1-\lambda_{j+1}^{2}\right)}{n-1}+\frac{\left(1-\lambda_{j+1}^{2}\right) \lambda_{j}^{2}\left(1-\lambda_{n}^{2}\right)}{2}+\frac{\left(1-\lambda_{j}^{2}\right) \lambda_{j+1}^{2}\left(1-\lambda_{n}^{2}\right)}{2}
$$

and

$$
A_{n-1}=\frac{\left(1-\lambda_{1}^{2}\right)\left(1-\lambda_{n-1}^{2}\right)}{n-1}+\frac{\left(1-\lambda_{1}^{2}\right) \lambda_{n-1}^{2}\left(1-\lambda_{n}^{2}\right)}{2}+\frac{\left(1-\lambda_{n-1}^{2}\right) \lambda_{1}^{2}\left(1-\lambda_{n}^{2}\right)}{2} .
$$

We can rewrite $A_{j}$ as

$$
\begin{aligned}
& \frac{2\left(1-\lambda_{j}^{2}-\lambda_{j+1}^{2}+\lambda_{j}^{2} \lambda_{j+1}^{2}\right)+(n-1)\left[\lambda_{j}^{2}+\lambda_{j+1}^{2}-2 \lambda_{j}^{2} \lambda_{j+1}^{2}-\lambda_{n}^{2} \lambda_{j+1}^{2}-\lambda_{n}^{2} \lambda_{j}^{2}+2 \lambda_{j}^{2} \lambda_{j+1}^{2} \lambda_{n}^{2}\right]}{2(n-1)} \\
= & \frac{2+(n-3) \lambda_{j}^{2}+(n-3) \lambda_{j+1}^{2}-2(n-2) \lambda_{j}^{2} \lambda_{j+1}^{2}+2(n-1) \lambda_{j}^{2} \lambda_{j+1}^{2} \lambda_{n}^{2}-(n-1) \lambda_{n}^{2}\left(\lambda_{j}^{2}+\lambda_{j+1}^{2}\right)}{2(n-1)} \\
= & \frac{2+(n-3)\left(\lambda_{j}-\lambda_{j+1}\right)^{2}+2(n-3) \lambda_{j} \lambda_{j+1}\left(1-\lambda_{j} \lambda_{j+1}\right)+2 \lambda_{j}^{2} \lambda_{j+1}^{2}\left(\lambda_{n}^{2}-1\right)}{2(n-1)} \\
+ & \frac{2(n-2) \lambda_{j}^{2} \lambda_{j+1}^{2} \lambda_{n}^{2}+2-(n-1) \lambda_{n}^{2}\left(\lambda_{j}^{2}+\lambda_{j+1}^{2}\right)}{2(n-1)} .
\end{aligned}
$$


Similarly, $A_{n-1}$ becomes

$$
\begin{aligned}
& \frac{2+(n-3)\left(\lambda_{1}-\lambda_{n-1}\right)^{2}+2(n-3) \lambda_{1} \lambda_{n-1}\left(1-\lambda_{1} \lambda_{n-1}\right)+2 \lambda_{1}^{2} \lambda_{n-1}^{2}\left(\lambda_{n}^{2}-1\right)}{2(n-1)} \\
+ & \frac{2(n-2) \lambda_{1}^{2} \lambda_{n-1}^{2} \lambda_{n}^{2}+2-(n-1) \lambda_{n}^{2}\left(\lambda_{1}^{2}+\lambda_{n-1}^{2}\right)}{2(n-1)} .
\end{aligned}
$$

Note that $A_{j} \geq 0$ and $A_{n-1} \geq 0$ if we require

$$
\left(\lambda_{j}^{2}+\lambda_{j+1}^{2}\right) \lambda_{n}^{2} \leq \frac{2}{n-1}+\left(2-\frac{2}{n-1}\right) \lambda_{j}^{2} \lambda_{j+1}^{2} \lambda_{n}^{2}
$$

and

$$
\left(\lambda_{1}^{2}+\lambda_{n-1}^{2}\right) \lambda_{n}^{2} \leq \frac{2}{n-1}+\left(2-\frac{2}{n-1}\right) \lambda_{1}^{2} \lambda_{n-1}^{2} \lambda_{n}^{2} .
$$

This completes the proof.

\section{Stability OF Minimal GRAPHS}

In this section, we obtain several criteria for the stability of minimal graphs in terms of the singular values of $d f$. These results are the direct application of convexity of the area functional.

Theorem 4.1. Let $M$ and $N$ be Riemannian manifolds and $\Sigma$ be the graph of a map $f: M \rightarrow N$. Suppose that the sectional curvature of $N$ is non-positive and $\Sigma$ is minimal in $M \times N$. Then $\Sigma$ is stable if $\lambda_{i}^{2} \lambda_{j}^{2} \leq 1$ for any $1 \leq i \neq j \leq n$, and

$$
1+\sum_{k=2}^{n}(k-1)(-1)^{k-1} \sum_{1 \leq j_{1}<j_{2}<\cdots<j_{k} \leq n} \lambda_{j_{1}}^{2} \cdots \lambda_{j_{k}}^{2} \geq 0
$$

or equivalently

$$
\prod_{i=1}^{n}\left(1-\lambda_{i}^{2}\right)+\sum_{i=1}^{n}\left(1-\lambda_{1}^{2}\right) \cdots \lambda_{i}^{2} \cdots\left(1-\lambda_{n}^{2}\right) \geq 0 .
$$

Proof. It suffices to show that the second variation $\left.\frac{d^{2} \mathcal{V}_{t}}{d t^{2}}\right|_{t=0}$ is non-negative. From Proposition 1] we have

$$
\begin{aligned}
\left.\frac{d^{2} \mathcal{V}_{t}}{d t^{2}}\right|_{t=0} \geq & \int_{M}\left(\sum_{i} \frac{1}{\left(1+\lambda_{i}^{2}\right)^{2}}\left\langle\nabla_{d f\left(a_{i}\right)} V, b_{i}\right\rangle^{2}\right. \\
& \left.+\sum_{i \neq j} \frac{\lambda_{i} \lambda_{j}}{\left(1+\lambda_{i}^{2}\right)\left(1+\lambda_{j}^{2}\right)}\left\langle\nabla_{d f\left(a_{i}\right)} V, b_{i}\right\rangle\left\langle\nabla_{d f\left(a_{j}\right)} V, b_{j}\right\rangle\right) d v \\
& +\int_{M}\left(\sum_{i \neq j} \frac{1}{\left(1+\lambda_{i}^{2}\right)\left(1+\lambda_{j}^{2}\right)}\left\langle\nabla_{d f\left(a_{i}\right)} V, b_{j}\right\rangle^{2}\right. \\
& \left.-\sum_{i \neq j} \frac{\lambda_{i} \lambda_{j}}{\left(1+\lambda_{i}^{2}\right)\left(1+\lambda_{j}^{2}\right)}\left\langle\nabla_{d f\left(a_{i}\right)} V, b_{j}\right\rangle\left\langle\nabla_{d f\left(a_{j}\right)} V, b_{i}\right\rangle\right) d v .
\end{aligned}
$$

The sum of the first two integrands on the right hand side of (4.1) is non-negative by Lemma 3.1, Theorem 3.2 and Theorem 3.4 by letting $w_{i}=\left\langle\nabla_{d f\left(a_{i}\right)} V, b_{i}\right\rangle$. While 
the sum of the last two integrands on the right hand side of (4.1), after symmetrizing the indices, can be written as

$$
\sum_{i \neq j} \frac{\left\langle\nabla_{d f\left(a_{i}\right)} V, b_{j}\right\rangle^{2}-2 \lambda_{i} \lambda_{j}\left\langle\nabla_{d f\left(a_{i}\right)} V, b_{j}\right\rangle\left\langle\nabla_{d f\left(a_{j}\right)} V, b_{i}\right\rangle+\left\langle\nabla_{d f\left(a_{j}\right)} V, b_{i}\right\rangle^{2}}{2\left(1+\lambda_{i}^{2}\right)\left(1+\lambda_{j}^{2}\right)},
$$

it is clearly non-negative since $\lambda_{i} \lambda_{j} \leq 1$ for $i \neq j$. Hence we have $\left.\frac{d^{2} \mathcal{V}_{t}}{d t^{2}}\right|_{t=0} \geq 0$ and the minimal submanifold is stable as claimed.

Remark 4.2. The theorem above implies that $\Sigma$ is stable whenever the area functional is convex with respect to its associated singular values.

From Corollary 1 and Remark 4.2, we recover the following theorem proved in LW2.

Theorem 4.3 ([LW2 $)$. Let $M$ and $N$ be Riemannian manifolds and $\Sigma$ be the graph of a map $f: M \rightarrow N$. Suppose the sectional curvature of $N$ is non-positive and $\Sigma$ is minimal in $M \times N$. Then $\Sigma$ is stable if $\lambda_{i}^{2} \leq 1$ for $1 \leq i \leq n$.

When $\operatorname{rank}(d f) \leq p$, the area functional only depends on the $p$ non-zero singular values, and it reduces to the $p$-dimensional case. Namely, if we denote the non-zero singular values of $d f$ to be $\left\{\lambda_{i}\right\}_{i=1}^{p}$, then we have

$$
\begin{aligned}
& 1+\sum_{k=2}^{n}(k-1)(-1)^{k-1} \sum_{1 \leq j_{1}<j_{2}<\cdots<j_{k} \leq n} \lambda_{j_{1}}^{2} \cdots \lambda_{j_{k}}^{2} \\
= & 1+\sum_{k=2}^{p}(k-1)(-1)^{k-1} \sum_{1 \leq j_{1}<j_{2}<\cdots<j_{k} \leq p} \lambda_{j_{1}}^{2} \cdots \lambda_{j_{k}}^{2} .
\end{aligned}
$$

Therefore, from Proposition 2 and Remark 4.2 we have

Theorem 4.4. Let $M$ and $N$ be Riemannian manifolds and $\Sigma$ be the graph of a map $f: M \rightarrow N$ with rank $(d f) \leq p$ for some integer $p>1$. Suppose that the sectional curvature of $N$ is non-positive and $\Sigma$ is minimal in $M \times N$. Then $\Sigma$ is stable if $\lambda_{i}^{2} \lambda_{j}^{2} \leq 1$ for any $1 \leq i \neq j \leq n$ and

$$
\left(\lambda_{i}^{2}+\lambda_{j}^{2}\right) \lambda_{k}^{2} \leq \frac{2}{p-1}+\left(2-\frac{2}{p-1}\right) \lambda_{i}^{2} \lambda_{j}^{2} \lambda_{k}^{2}
$$

for any $1 \leq i \neq j \neq k \leq n$.

From Theorem 4.4, we can improve the condition in Theorem 4.1 of [LW2] from $\left|\bigwedge^{2} d f\right| \leq \frac{1}{p-1}$ to $\left|\bigwedge^{2} d f\right| \leq \frac{1}{\sqrt{p-1}}$.

Theorem 4.5. Let $M$ and $N$ be Riemannian manifolds and $\Sigma$ be the graph of a map $f: M \rightarrow N$ with $\operatorname{rank}(d f) \leq p$ for some integer $p>1$. Suppose that the sectional curvature of $N$ is non-positive and $\Sigma$ is minimal in $M \times N$. Then $\Sigma$ is stable if $\left|\bigwedge^{2} d f\right| \leq \frac{1}{\sqrt{p-1}}$.

Proof. The condition $\left|\bigwedge^{2} d f\right| \leq \frac{1}{\sqrt{p-1}}$ implies $\left(\lambda_{i}^{2}+\lambda_{j}^{2}\right) \lambda_{k}^{2} \leq \frac{2}{p-1}$ for any $1 \leq i \neq$ $j \neq k \leq n$. Thus (4.3) holds and the result follows from Theorem 4.4.

In the following, we prove another stability result in terms of the area functional which improves a similar result in [LW1. 
Theorem 4.6. Let $M$ and $N$ be Riemannian manifolds and $\Sigma$ be the graph of a map $f: M \rightarrow N$. Suppose the sectional curvature of $N$ is non-positive and $\Sigma$ is minimal in $M \times N$. Then $\Sigma$ is stable if $\prod_{i=1}^{n}\left(1+\lambda_{i}^{2}\right) \leq 4$.

Proof. It suffices to prove that $\lambda_{i} \lambda_{j} \leq 1$ for any $1 \leq i \neq j \leq n$ and

$$
\prod_{i=1}^{n}\left(1-\lambda_{i}^{2}\right)+\sum_{i=1}^{n}\left(1-\lambda_{1}^{2}\right) \cdots \lambda_{i}^{2} \cdots\left(1-\lambda_{n}^{2}\right) \geq 0 .
$$

From Lemma 2.2, the condition $\lambda_{i}^{2} \lambda_{j}^{2} \leq 1$ for any $1 \leq i \neq j \leq n$ is implied by $\prod_{i=1}^{n}\left(1+\lambda_{i}^{2}\right) \leq 4$. By Remark 3.3, we just need to consider the case where

$$
0 \leq \lambda_{1}^{2} \leq \cdots \leq \lambda_{n-1}^{2}<1<\lambda_{n}^{2} .
$$

We will prove (4.4) by induction. When $n=2$, equation (4.4) clearly holds since

$$
\begin{aligned}
& \prod_{i=1}^{2}\left(1-\lambda_{i}^{2}\right)+\sum_{i=1}^{n}\left(1-\lambda_{1}^{2}\right) \cdots \lambda_{i}^{2} \cdots\left(1-\lambda_{n}^{2}\right) \\
= & \left(1-\lambda_{1}^{2}\right)\left(1-\lambda_{2}^{2}\right)+\lambda_{1}^{2}\left(1-\lambda_{2}^{2}\right)+\left(1-\lambda_{1}^{2}\right) \lambda_{2}^{2}=1-\lambda_{1}^{2} \lambda_{2}^{2} .
\end{aligned}
$$

Note that

$$
\prod_{i=1}^{n}\left(1-\lambda_{i}^{2}\right)+\sum_{i=1}^{n}\left(1-\lambda_{1}^{2}\right) \cdots \lambda_{i}^{2} \cdots\left(1-\lambda_{n}^{2}\right)=\prod_{l=1}^{n}\left(1-\lambda_{l}^{2}\right)\left(1+\sum_{j=1}^{n} \frac{\lambda_{j}^{2}}{1-\lambda_{j}^{2}}\right) .
$$

Let $x=\left(x_{1}, \cdots, x_{n}\right)$,

$$
\mathcal{F}(x)=\prod_{l=1}^{n}\left(1-x_{l}^{2}\right)\left(1+\sum_{j=1}^{n} \frac{x_{j}^{2}}{1-x_{j}^{2}}\right), \quad G(x)=\prod_{i=1}^{n}\left(1+x_{i}^{2}\right)
$$

and $\Omega=\left\{\left(x_{1}, \cdots, x_{n}\right) \mid G(x) \leq 4\right\}$. Inequality (4.4) is equivalent to $\mathcal{F}(x) \geq 0$ in $\Omega$.

Since $\Omega$ is a compact set, $\mathcal{F}$ must achieve its extremal value in $\Omega$. It suffices to show that $\mathcal{F}(x) \geq 0$ in $\operatorname{int}(\Omega)$ and $\mathcal{F}(x) \geq 0$ subject to the constraint $G(x)=4$. Consider the Lagrangian $\mathcal{L}(x)=\mathcal{F}-\lambda G$. First, we compute

$$
\begin{aligned}
& \frac{\partial \mathcal{F}}{\partial x_{i}}=\frac{\partial}{\partial x_{i}} \prod_{l=1}^{n}\left(1-x_{l}^{2}\right)\left(1+\sum_{k=1}^{n} \frac{x_{k}^{2}}{1-x_{k}^{2}}\right) \\
= & -2 x_{i} \prod_{l \neq i}\left(1-x_{l}^{2}\right)\left(1+\sum_{k=1}^{n} \frac{x_{k}^{2}}{1-x_{k}^{2}}\right)+\prod_{l=1}^{n}\left(1-x_{l}^{2}\right) \frac{2 x_{i}}{\left(1-x_{i}^{2}\right)^{2}} \\
= & 2 x_{i} \prod_{l=1}^{n}\left(1-x_{l}^{2}\right)\left[-\frac{1}{1-x_{i}^{2}}-\frac{1}{1-x_{i}^{2}}\left(\sum_{k=1}^{n} \frac{x_{k}^{2}}{1-x_{k}^{2}}\right)+\frac{1}{\left(1-x_{i}^{2}\right)^{2}}\right] \\
= & 2 x_{i} \prod_{l=1}^{n}\left(1-x_{l}^{2}\right)\left[\frac{-1+x_{i}^{2}+1-x_{i}^{2}}{\left(1-x_{i}^{2}\right)^{2}}-\frac{1}{1-x_{i}^{2}}\left(\sum_{k \neq i} \frac{x_{k}^{2}}{1-x_{k}^{2}}\right)\right] \\
= & 2 x_{i} \prod_{l=1}^{n}\left(1-x_{l}^{2}\right)\left[-\frac{1}{1-x_{i}^{2}}\left(\sum_{k \neq i} \frac{x_{k}^{2}}{1-x_{k}^{2}}\right)\right] \\
= & -2 x_{i}\left[\prod_{l \neq i}\left(1-x_{l}^{2}\right)\right] \sum_{k \neq i} \frac{x_{k}^{2}}{1-x_{k}^{2}}
\end{aligned}
$$


and

$$
\frac{\partial G}{\partial x_{i}}=\frac{\partial}{\partial x_{i}} \prod_{l=1}^{n}\left(1+x_{l}^{2}\right)=2 x_{i} \prod_{l \neq i}\left(1+x_{l}^{2}\right) .
$$

The first order condition $\nabla \mathcal{L}=0$ requires that

$$
\frac{\partial \mathcal{L}}{\partial x_{i}}=-2 x_{i}\left[\prod_{l \neq i}\left(1-x_{l}^{2}\right)\right] \sum_{k \neq i} \frac{x_{k}^{2}}{1-x_{k}^{2}}-2 x_{i} \lambda \prod_{l \neq i}\left(1+x_{l}^{2}\right)=0 .
$$

Note that $F(x) \geq 0$ if $x_{i}=0$ for some $i$, since it will be reduced to one dimension less and the result holds by induction. So we may assume in the following that $x_{i} \neq 0$, and $x_{i}^{2} \neq 1$ for $1 \leq i \leq n$ by (4.5). Thus $\frac{\partial \mathcal{L}}{\partial x_{i}}=0$ implies that

$$
\sum_{l \neq i} \frac{x_{l}^{2}}{1-x_{l}^{2}}+\lambda \prod_{l \neq i} \frac{1+x_{l}^{2}}{1-x_{l}^{2}}=0 \text { for } 1 \leq i \leq n .
$$

There are two cases to be discussed.

Case 1: $\lambda=0$. This corresponds to the case when $F$ achieves an interior extremal value. It leads to

$$
\sum_{l \neq i} \frac{x_{l}^{2}}{1-x_{l}^{2}}=0 \text { for } 1 \leq i \leq n .
$$

The difference of any two equations in (4.8) gives

$$
0=\sum_{l \neq i} \frac{x_{l}^{2}}{1-x_{l}^{2}}-\sum_{l \neq j} \frac{x_{l}^{2}}{1-x_{l}^{2}}=\frac{x_{j}^{2}-x_{i}^{2}}{\left(1-x_{i}^{2}\right)\left(1-x_{j}^{2}\right)}
$$

for any $1 \leq i \neq j \leq n$, which implies $x_{1}^{2}=x_{2}^{2}=\cdots=x_{n}^{2}$. It follows that $x_{1}^{2}=x_{2}^{2}=\cdots=x_{n}^{2}=0$ by (4.8) and $F(0, \cdots, 0)=1>0$.

Case 2: $\lambda \neq 0$ and $\prod_{i=1}^{n}\left(1+x_{i}^{2}\right)=4$. Let $\left(y_{1}, y_{2}, \cdots, y_{n}\right)$ be a solution to the system (4.7) subject to the constraint $\prod_{i=1}^{n}\left(1+y_{i}^{2}\right)=4$. By (4.5) we just need to consider the case

$$
0 \leq y_{1}^{2} \leq y_{2}^{2} \leq \cdots \leq y_{n-1}^{2}<1<y_{n}^{2}
$$

The difference of any two equations in (4.7) gives

$$
\begin{aligned}
0 & =\left[\sum_{l \neq i} \frac{y_{l}^{2}}{1-y_{l}^{2}}+\lambda \prod_{l \neq i} \frac{1+y_{l}^{2}}{1-y_{l}^{2}}\right]-\left[\sum_{l \neq j} \frac{y_{l}^{2}}{1-y_{l}^{2}}+\lambda \prod_{l \neq j} \frac{1+y_{l}^{2}}{1-y_{l}^{2}}\right] \\
& =\left(\frac{y_{j}^{2}}{1-y_{j}^{2}}-\frac{y_{i}^{2}}{1-y_{i}^{2}}\right)+\lambda\left(\prod_{l \neq i, j} \frac{1+y_{l}^{2}}{1-y_{l}^{2}}\right)\left(\frac{1+y_{j}^{2}}{1-y_{j}^{2}}-\frac{1+y_{i}^{2}}{1-y_{i}^{2}}\right) \\
& =\frac{y_{j}^{2}-y_{i}^{2}}{\left(1-y_{i}^{2}\right)\left(1-y_{j}^{2}\right)}\left(1+2 \lambda \prod_{l \neq i, j} \frac{1+y_{l}^{2}}{1-y_{l}^{2}}\right) .
\end{aligned}
$$

Using (4.9) and applying the above equation to $1 \leq i \leq n-1$ and $j=n$, we obtain

$$
1+2 \lambda \prod_{l \neq i, n} \frac{1+y_{l}^{2}}{1-y_{l}^{2}}=0 \text { for } 1 \leq i \leq n-1 .
$$


Plugging it into (4.7), we have

$$
\begin{aligned}
0 & =\sum_{l \neq i, n} \frac{y_{l}^{2}}{1-y_{l}^{2}}+\frac{y_{n}^{2}}{1-y_{n}^{2}}+\left(\lambda \prod_{l \neq i, n} \frac{1+y_{l}^{2}}{1-y_{l}^{2}}\right) \cdot \frac{1+y_{n}^{2}}{1-y_{n}^{2}} \\
& =\sum_{l \neq i, n} \frac{y_{l}^{2}}{1-y_{l}^{2}}+\frac{y_{n}^{2}}{1-y_{n}^{2}}-\frac{1}{2} \cdot \frac{1+y_{n}^{2}}{1-y_{n}^{2}} \\
& =\sum_{l \neq i, n} \frac{y_{l}^{2}}{1-y_{l}^{2}}-\frac{1}{2} .
\end{aligned}
$$

Hence

$$
\sum_{l \neq i, n} \frac{y_{l}^{2}}{1-y_{l}^{2}}=\frac{1}{2} \quad \text { for } 1 \leq i \leq n-1 .
$$

The difference of any two equations in (4.10) leads to $y_{1}^{2}=y_{2}^{2}=\cdots=y_{n-1}^{2}$. It follows that $y_{1}^{2}=y_{2}^{2}=\cdots=y_{n-1}^{2}=\frac{1}{2 n-3}<1$ from (4.10), and we have $y_{n}^{2}=$ $4\left(\frac{2 n-3}{2 n-2}\right)^{n-1}-1$ using $\prod_{l=1}^{n}\left(1+y_{l}^{2}\right)=4$. Note that $y_{n}^{2}>1$ since $\left(1-\frac{1}{2 n-2}\right)^{2 n-2}>\frac{1}{4}$ for $n \geq 3$. Thus we have $\prod_{l=1}^{n}\left(1-y_{l}^{2}\right)<0$ and

$$
\begin{aligned}
1+\sum_{j=1}^{n} \frac{y_{j}^{2}}{1-y_{j}^{2}} & =1+(n-1) \frac{y_{1}^{2}}{1-y_{1}^{2}}+\frac{y_{n}^{2}}{1-y_{n}^{2}} \\
& =\frac{n-1}{2 n-4}+\frac{1}{1-y_{n}^{2}} \\
& =\frac{(n-1)\left(1-y_{n}^{2}\right)+2 n-4}{(2 n-4)\left(1-y_{n}^{2}\right)} \\
& =\frac{(4 n-6)-(4 n-4)\left(\frac{2 n-3}{2 n-2}\right)^{n-1}}{(2 n-4)\left(1-y_{n}^{2}\right)} \\
& =\frac{(4 n-4)\left(\frac{2 n-3}{2 n-2}-\left(\frac{2 n-3}{2 n-2}\right)^{n-1}\right)}{(2 n-3)\left(1-y_{n}^{2}\right)} \\
& <0 .
\end{aligned}
$$

Therefore $F(y)=\prod_{l=1}^{n}\left(1-y_{l}^{2}\right)\left(1+\sum_{j=1}^{n} \frac{y_{j}^{2}}{1-y_{j}^{2}}\right)>0$, and it completes the proof of the theorem.

\section{ACKNOWLEDGEMENTS}

Part of this paper was completed while the second author was visiting Taida Institute of Mathematical Sciences (TIMS) and the National Center for Theoretical Sciences, Taipei Office in National Taiwan University, Taipei, Taiwan. The second author wishes to express his gratitude for the excellent support provided by both centers during his stay. The research was partially supported by a Collaboration Grant for Mathematicians from the Simons Foundation (\#239677 to the second author). Both authors would like to thank Mu-Tao Wang for many enlightening discussions and his interest in this work. 


\section{REFERENCES}

[LO] H. B. Lawson Jr. and R. Osserman, Non-existence, non-uniqueness and irregularity of solutions to the minimal surface system, Acta Math. 139 (1977), no. 1-2, 1-17, DOI 10.1007/BF02392232. MR 452745 (80b:35059)

[LW1] Yng-Ing Lee and Mu-Tao Wang, A stability criterion for nonparametric minimal submanifolds, Manuscripta Math. 112 (2003), no. 2, 161-169, DOI 10.1007/s00229-003-0404-2. MR2064913 (2005b:53096)

[LW2] Yng-Ing Lee and Mu-Tao Wang, A note on the stability and uniqueness for solutions to the minimal surface system, Math. Res. Lett. 15 (2008), no. 1, 197-206. MR2367184 (2008m:53016)

[OS] Robert Osserman, Minimal varieties, Bull. Amer. Math. Soc. 75 (1969), 1092-1120. MR.0276875 (43 \#2615)

Department of Mathematics, National Taiwan University, Taipei, Taiwan - and National Center for Theoretical Sciences, Taipei Office, Taipei, Taiwan

E-mail address: yilee@math.ntu.edu.tw

Department of Mathematics and Statistics, University of Toledo, Toledo, Ohio 43606

E-mail address: Mao-Pei.Tsui@Utoledo.edu 